\title{
Die Bedeutung der GOZ für das Einkommen der Zahnärzte
}

\section{Eine Umsatz- und Kostenanalyse zur bevorstehenden GOZ-Novelle}

\section{Frank Schulze Ehring}

Dr. Frank Schulze Ehring ist Leiter des Referats Grundsatzfragen der Gesundheitspolitik bei der PKV in Berlin

Bei der Reform der Gebührenordnung für Zahnärzte (GOZ) ist darauf zu achten, dass die GOZ nicht zum "Kostentreiber" wird. Die Versicherten sind vor Mehrbelastungen zu schützen. Die zu schützenden Versicherten sind sowohl Versicherte der PKV als auch der GKV. Denn regelmäßig stellt der Zahnarzt neben Privatversicherten auch Selbstzahlern eine Rechnung nach der GOZ. Von diesen Selbstzahlern gibt es immer mehr. Spätestens nämlich seit es in der GKV beim Zahnersatz nur noch einen Festzuschuss gibt, hat sich die GKV im Bereich der Zahnleistungen zur Teilkaskoversicherung entwickelt. Im Interesse von 80 Millionen Versicherten in Deutschland sind deshalb bei der Reform der GOZ die Kostenentwicklungen zu berücksichtigen. So steht es auch im Koalitionsvertrag. Das sollte Anlass genug sein, einen Blick auf die tatsächliche Ausgaben- und Einkommensentwicklungen in der privatzahnärztlichen Versorgung zu werfen.

\section{Aktueller Hintergrund}

Der ehemalige Bundesgesundheitsminister Phillip Rösler vertrat bei Amtsantritt die Auffassung, »nicht den Ärzten oder anderen Leistungserbringern ", 1 sondern den Menschen verpflichtet zu sein. Er sei - so der ehemalige Gesundheitsminister - insbesondere »Anwalt der Versicherten ${ }^{2}{ }^{2}$ Mit diesem Selbstverständnis sollte auch für den neuen Gesundheitsminister Daniel Bahr einhergehen, dass für alle Krankenversicherten in Deutschland die Bezahlbarkeit der zahnärztlichen Leistungen langfristig gewährleistet bleibt. Entsprechend ist darauf $\mathrm{zu}$ achten, dass die von der Bundesregierung in der laufenden Legislaturperiode beabsichtigte Reform der Gebührenordnung für Zahnärzte (GOZ) nicht zum »Kostentreiber" wird. Die Versicherten sind vor übermäßigen Belastungen zu schützen. Kostenentwicklungen sind bei der Reform der GOZ - so steht es auch im Koalitionsvertrag - zu berücksichtigen.

Die zu schützenden Versicherten sind im Fall der geplanten Novellierung der
GOZ sowohl Versicherte der PKV als auch der GKV. Denn auch wenn die Leistungen im Rahmen des Sachleistungsprinzips der GKV nicht nach der GOZ, sondern nach dem Bewertungsmaßstab zahnärztlicher Leistungen (BEMA) vergütet werden, stellt der Zahnarzt neben PKV-Versicherten in der Voll- und Zusatzversicherung auch gesetzlich versicherten Selbstzahlern (partiell) eine Rechnung nach der GOZ. Von diesen Selbstzahlern gibt es immer mehr. Spätestens nämlich seit es in der GKV beim Zahnersatz nicht mehr eine prozentuale Erstattung auf die Behandlungskosten, sondern einen Festzuschuss je Indikation gibt (2005), hat sich die GKV im Bereich der Zahnleistungen zur Teilkaskoversicherung entwickelt. Alle gesetzlich Versicherten sind anteilig mehr und mehr zum Selbstzahler geworden. Von den Konsequenzen der Novellierung der GOZ

1 Vgl. Welt, 28.11.2009.

2 Vgl. General Anzeiger, 20.3.2010. 
sind deshalb 80 Millionen Menschen betroffen.

Vor dem Hintergrund, dass die Leistungsausgaben der privaten Krankenversicherung für Zahnbehandlung seit Jahren überproportional zur Preisentwicklung steigen, muss die bevorstehende Novellierung der GOZ kostenneutral sein. Es gibt keinen Nachholbedarf bei der Honorierung privatzahnärztlicher Leistung. Das ist eine Feststellung, mit der man häufig zum Teil sehr heftige, in der Regel ab-

\section{Dabei fällt auf, dass alle Ergebnisse in ein und dieselbe Richtung weisen}

lehnende Reaktionen in der Zahnärzteschaft auslöst. Diese Entwicklung sollte Anlass genug sein, einen ausführlichen und genauen Blick auf die Ausgabenentwicklung in der PKV zu werfen. Im Folgenden soll differenziert auf die Ausgabenentwicklung in der zahnärztlichen Versorgung eingegangen und die Bedeutung der Privatpatienten und Selbstzahler für den Umsatz beziehungsweise das Einkommen der Zahnärzte betrachtet werden. Dabei lohnt es sich, die Ursachen der Ausgaben- bzw. Umsatzentwicklung - getrennt nach Selbstzahlereffekt, Preis-, Struktur- und Mengeneffekt - zu thematisieren.

Alle folgenden Zahlen und Hinweise zur Entwicklung der zahnärztlichen Ausgaben in der PKV auf der einen und zur Entwicklung der Umsätze beziehungsweise Einkommen der Zahnärzte auf der anderen Seite werden aus Daten des PKV-Verbandes, des Wissenschaftlichen Instituts der PKV (WIP) und aus Daten des Bundesgesundheitsministeriums für Gesundheit (BMG) generiert. Auswertungen des Statistischen Bundesamtes und der Kassenzahnärztlichen Bundesvereinigung (KZBV) ergänzen diese Zahlen. Dabei fällt auf, dass - unabhängig von der originären Datenquelle - alle Ergebnisse in ein und dieselbe Richtung weisen: Die Bedeutung der Privatpatienten für die Umsätze und das Einkommen der Zahnärzte wächst. Die unter anderem von den Leistungserbringern selbst verursachte Ausgabenund Umsatzdynamik muss sowohl im
Interesse der Privatversicherten, der Selbstzahler als auch der Zahnärzteschaft gestoppt werden.

\section{Ausgabenentwicklung in der zahnärztlichen Versorgung}

\subsection{Ergebnisse auf Basis der Ausgabenstatistik von PKV und GKV}

Im Jahr 1999 betrugen die Gesamtausgaben der PKV in der zahnärztlichen Versorgung 1,79 Mrd. €. Im Jahr 2009 waren es schon 3,04 Mrd. €. ${ }^{3}$ Dieser Anstieg, der einem Wachstum von fast $70 \%$ innerhalb von 10 Jahren entspricht, deutet schon auf den ersten Blick auf eine problematische Entwicklung in der zahnärztlichen Vergütung hin. Dabei muss allerdings zweifellos darauf hingewiesen werden, dass - weil die Zahl der Versicherten in der PKV angestiegen ist - die zahnärztlichen Ausgaben je Versicherten einen besseren methodischen Ausgangspunkt zur Bewertung der Ausgabenentwicklung in der PKV bieten.

\subsubsection{Ausgabenentwicklung je Versicherten}

Ohne die Leistungen der Beihilfe und Selbstbehalte zu berücksichtigen, werden im Zahlenbericht 2009/2010 die Ausgaben der PKV für die zahnärztliche Arztbehandlung mit $348,3 €$ je Vollversicherten (2009) ausgewiesen. Ein Jahrzehnt zuvor lagen diese Ausgaben noch bei $245,3 €{ }^{4}$ Das entspricht einem Wachstum von $42 \%$.

Die Betrachtung der PKV-Ausgaben je Versicherten greift allerdings $\mathrm{zu}$ kurz. Es ist unbedingt zuberücksichtigen, dass heute fast die Hälfte der Privatversicherten beihilfeberechtigt sind $(47,6 \%)$ und nur einen Teil ihrer zahnärztlichen Ausgaben von der PKV erstattet bekommen. Die methodische Berücksichtigung dieses Tatbestands setzt voraus, dass für alle Beihilfeberechtigten, deren Beihilfeanteil in der Regel zwischen 50 und $80 \%$ liegt, ein gewichteter durchschnittlicher Beihilfeanteil gebildet werden kann. Das ist mit nicht unerheblichen Schwierigkei-

\section{Abb. 1:}

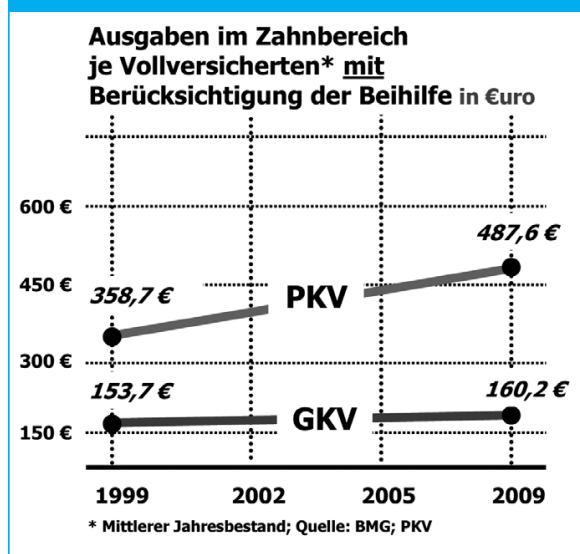

ten verbunden, unter anderem weil sich in einer Zeitraumbetrachtung nicht nur die Besetzungen der Beihilfestufen, sondern auch die Beihilfevorschriften ständig ändern. In der PKV liegen diesbezüglich keine aggregierten Informationen vor. Vereinfacht wird deshalb im Folgenden angenommen, dass im Durchschnitt alle Beihilfeberechtigen mit einem Anteil von $40 \%$ in der privaten Krankenversicherung versichert sind.

Um die Quotenversicherung (40\%) der Beihilfeberechtigten in der privaten Krankenversicherung zu berücksichtigen, ist die Zahl der Beihilfeberechtigten mit dem Faktor 0,4 gewichtet worden. Das Ergebnis: Unter Berücksichtigung des Beihilfeanteils der Beihilfeberechtigten in der PKV sind im Zeitraum 1999 bis 2009 die Ausgaben in der zahnärztlichen Behandlung je Vollversicherten von $358,7 €$ auf $487,6 €$ angestiegen. Das entspricht einem Wachstum von 35,9 \%.5 Die Inflation lag zwischen 1999 und 2009 dagegen bei lediglich $17,1 \%$. In gleicher Zeit sind die Ausgaben der gesetzlichen Krankenversicherung je Versicherten von $153,7 €(1999)$ auf nur 160,2 € im Jahr 2009 (+4,3\%) ange-

3 Vgl. PKV-Zahlenberichte (1999/2000) und (2009/2010).

4 Die Zahlen beziehen sich jeweils auf den mittleren Versichertenjahresbestand.

5 Das Wachstum unter Berücksichtigung der gewichteten Beihilfeberechtigten liegt unter anderem deshalb unter dem Wachstum ohne Berücksichtigung der gewichteten Beihilfeberechtigen, weil in den letzten 10 Jahren der Anteil der Privatversicherten mit Beihilfeberechtigung an allen Versicherten von $52,7 \%$ (1999) auf 47,6 \% (2009) gesunken ist. Die absoluten ProKopf-Ausgaben in liegen unter Einbezug der Beihilfe natürlich immer über denen der Ausgaben ohne Beihilfe. 
stiegen. ${ }^{6}$ Dabei darf aber nicht unerwähnt bleiben, dass im Zahnbereich in der GKV viele Leistungskürzungen stattgefunden haben. Ein Wachstumsvergleich sollte demnach in erster Linie auf den Vergleich zwischen der PKV und der allgemeinen Preisentwicklung beschränkt bleiben.

\subsubsection{Ausgabenanteil der zahnärztlichen Versorgung}

Ergänzend zur Pro-Kopf-Analyse lässt sich der Ausgabenanteil der Zahnarztbehandlung an den Gesamtausgaben der PKV betrachten. Dabei stellt man fest, dass der Ausgabenanteil für zahnärztliche Leistungen an den Gesamtausgaben der Krankenversicherung in der PKV in den letzten Jahren nicht konstant geblieben ist. Während in der GKV der Anteil der Ausgaben im Bereich der zahnärztlichen Versorgung an den Gesamtausgaben von 8,9 \% im Jahr 1999 auf inzwischen 7,0 \% (2009) gesunken ist, ${ }^{7}$ ist der Anteil in der PKV in 10 Jahren um 0,7 Prozentpunkte von $14,2 \%$ auf $14,9 \%$ angestiegen. ${ }^{8}$ Dabei gilt es stets zu berücksichtigen, dass die große Bezugsgröße »Ausgaben insgesamt « eine enorme Hebelwirkung entfaltet. Dem Anstieg der Quote entsprechen Mehrausgaben von 1,25 Mrd. €.

Bei einer Erweiterung des Beobachtungszeitraums ist Folgendes festzustellen: Während im Jahr 2009 der Ausgabenanteil der zahnärztlichen Versor-

Tab. 1: Durchschnittlicher Umsatz je Zahnarzt durch PKV-Versicherte (ohne Berücksichtigung von Beihilfe + Selbstbehalte)

\begin{tabular}{|l|l|l|l|l|l|}
\hline & 2001 & 2004 & 2006 & 2009 & $\begin{array}{l}\text { Verände- } \\
\text { rung in \% } \\
(2001- \\
2009)\end{array}$ \\
\hline $\begin{array}{l}\text { Zahnleistun- } \\
\text { gen }\end{array}$ & 2,03 Mrd. $€$ & 2,32 Mrd. € & 2,50 Mrd. $€$ & 3,04 Mrd. $€$ & $+49,8 \%$ \\
\hline $\begin{array}{l}\text { Zahl der } \\
\text { Zahnärzte }\end{array}$ & 60.858 & 62.146 & 62.534 & 64.287 & $+5,6 \%$ \\
\hline $\begin{array}{l}\text { Umsatz je } \\
\text { Zahnarzt }\end{array}$ & $33.356 €$ & $37.331 €$ & $39.978 €$ & $47.279 €$ & $+41,7 \%$ \\
\hline $\begin{array}{l}\text { Inflations- } \\
\text { index }\end{array}$ & 94,5 & 98,5 & 101,6 & 107,0 & $+13,2 \%$ \\
\hline $\begin{array}{l}\text { Quelle: PKV-Zahlenberichte; Statistisches Bundesamt; } \\
\text { Gesundheitsberichterstattung des Bundes; BZÄK }\end{array}$ & \\
\hline
\end{tabular}

gung 14,9 \% betrug, lag im Jahr 1986 der Anteil der zahnärztlichen Leistungen an den Gesamtausgaben noch bei $12,3 \% .^{9}$ Das entspricht einem Anstieg um 2,6 Prozentpunkte. Fazit: Von einem konstanten Anteil der zahnärztlichen Leistungen an den Gesamtausgaben kann keine Rede sein.

\subsubsection{Umsätze der Zahnärzte durch Privatversicherte}

Entsprechend der bis jetzt analysierten Entwicklungen der zahnärztlichen Versicherungsleistungen ist auch das Wachstum der Umsätze der Zahnärzte aus der privatärztlichen Vergütung überproportional. Das zeigt sich im Folgenden zunächst einmal ohne Berücksichtigung

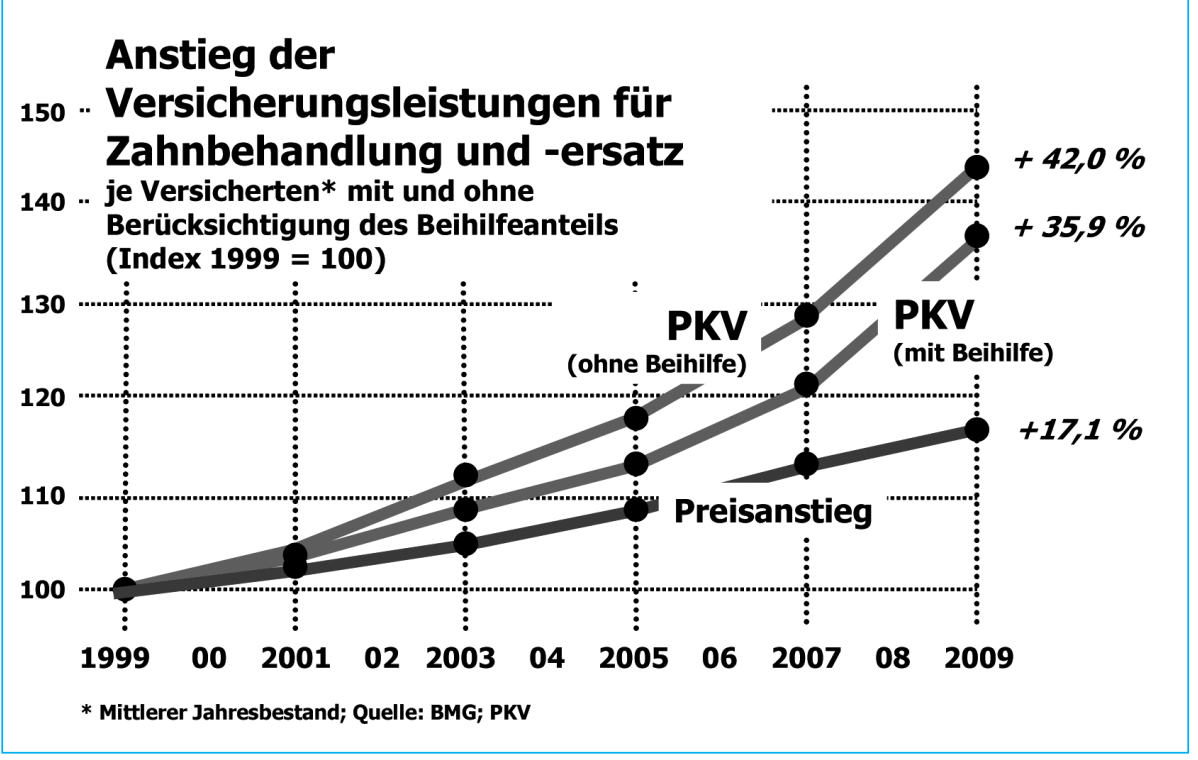

der Zahlungen der unterschiedlichen Beihilfeträger in Bund, Ländern und Kommunen.

Die im PKV-Zahlenbericht veröffentlichten Leistungen für die Zahnarztbehandlung enthalten keine Leistungen der Beihilfe. Sie beziehen sich ausschließlich auf Leistungen der PKV. Laut Zahlenbericht wurden im Jahr 2009 3,04 Mrd. € für die zahnärztliche Versorgung ausgegeben. ${ }^{10}$ Teilt man diese Zahlungen auf die 64.287 im Jahr 2009 im zahnärztlichen Bereich berufstätigen Zahnärzte (inklusive der in Zahnarztpraxen tätigen Assistenten und angestellten Zahnärzten) auf, ${ }^{11}$ ergibt sich ein durchschnittlicher Umsatz von ca. $47.279 €$ je Zahnarzt. Im Jahr 2001 lag die Zahl der berufstätigen Zahnärzte noch bei 60.858 . Auf diese entfielen PKV-Leistungen in Höhe von 2,03 Mrd. €. ${ }^{12}$ Es ergibt sich ein durchschnittlicher Umsatz im Jahr 2001 von ca. $33.356 €$. Zwischen 2001 und 2009 ist damit der durchschnittliche Umsatz durch privat versicherte Privatpatienten je tätigem Zahnarzt um fast $42 \%$ angestiegen. Die Inflation lag in dieser Zeit bei nur $13,2 \%$.

6 Vgl. GKV-Rechnungsergebnisse (endgültig), 1999 bis 2009.

7 Vgl. GKV-Rechnungsergebnisse (endgültig), 1999 bis 2009.

8 Vgl. PKV-Zahlenberichte (1999/2000) und (2009/2010).

9 Vgl. PKV-Zahlenbericht (1985/1986). 10 Vgl. PKV-Zahlenbericht (1999/2000).

11 Angaben der Bundeszahnärztekammer 2010.

12 Vgl. PKV-Zahlenbericht (2001/2002). 
Der hier vorgenommene zeitliche Umsatzvergleich geht davon aus, dass dem ausgewiesenen Umsatz immer eine gleichbleibende zeitliche Zahnarztleistung zugrunde liegt. Das ist mitnichten der Fall, wenn man die sogenannte Feminisierung des Zahnarztberufes berücksichtigen würde. ${ }^{13}$ Mit einem steigenden Anteil von Zahnärztinnen im niedergelassenen Bereich geht nämlich immer auch eine höhere Zahl von Teilzeit oder anderen zeitlichen Einschränkungen einher. Im Umkehrschluss heißt das, dass dem steigenden Umsatz je Zahnarzt eine stetig sinkende Leistung - gemessen in Zeiteinheiten (Zahnarztstunden) - zu Grunde liegt. Der oben skizzierte Anstieg des Umsatzes je Zahnarzt müsste entsprechend bereinigt werden. Er würde im Vergleich zu den oben genannten Zahlen je (standardisierter) Zeiteinheit erheblich ausgeprägter ausfallen.

Den skizzierten Beobachtungen zur Entwicklung der Umsätze der Zahnärzte könnte man kritisch entgegnen, dass sich die Erlöse direkt aus den Leistungsausgaben der PKV ergeben und damit Schadensregulierungskosten enthalten sind. Dabei gilt es allerdings zu berücksichtigen, dass die Kosten der Rechnungsprüfung entscheidend dazu

Tab. 2: Durchschnittlicher Umsatz je Zahnarzt durch Privatpatienten (ohne Berücksichtigung von Beihilfe + Selbstbehalte)

\begin{tabular}{|l|l|l|l|l|l|}
\hline & 2001 & 2003 & 2006 & 2008 & $\begin{array}{l}\text { Verände- } \\
\text { rung in \% } \\
(2001- \\
2006)\end{array}$ \\
\hline Leistungen & 2,79 Mrd. $€$ & 3,23 Mrd. $€$ & 3,08 Mrd. $€$ & 3,62 Mrd. $€$ & $+29,7 \%$ \\
\hline $\begin{array}{l}\text { Zahl der } \\
\text { Zahnärzte }\end{array}$ & 60.858 & 61.730 & 62.534 & 63.532 & $+4,4 \%$ \\
\hline $\begin{array}{l}\text { Umsatz je } \\
\text { Arzt }\end{array}$ & $45.844 €$ & $52.325 €$ & $49.253 €$ & $56.979 €$ & $+24,3 \%$ \\
\hline $\begin{array}{l}\text { Inflations- } \\
\text { index }\end{array}$ & 94,5 & 96,9 & 101,6 & 106,6 & $+12,8 \%$ \\
\hline $\begin{array}{l}\text { Ouelle: Ergebnisse aus der Mehrumsatzstudie des WIP; } \\
\text { Gesundheitsberichterstattung des Bundes; BZÄK }\end{array}$ & & & & \\
\hline
\end{tabular}

Ausgabendynamik wider. Erstens: Viele von Zahnärzten erbrachte Leistungen fallen unter die in der PKV weit verbreiteten Selbstbehalte. Diese Leistungen werden von den Versicherten nicht zur Kostenerstattung bei den Versicherungsunternehmen eingereicht. Konsequenterweise sind diese Leistungen nicht in der Ausgabenstatistik der PKV enthalten. Zweitens: Leistungen, die die Beihilfestellen der Beihilfeberechtigten übernehmen, sind bisher lediglich mittels einer durchschnittlichen Gewichtung der Beihilfeberechtigten hochgerechnet worden. Gerade weil sich die Beihilfevorschriften kontinuierlich verändert haben, kann das $\mathrm{m}$ at he mat is che Hochrechnen der Beihilfeleistungen

beitragen, dass gebührenrechtlich nicht korrekte Abrechnungen gekürzt werden. Ohne eine systematische Rechnungsprüfung hätten sich die ambulanten Leistungsausgaben in der privaten Krankenversicherung noch wesentlich dynamischer entwickelt.

\subsection{Ergebnisse auf Basis der Forschung des Wissenschaftlichen Instituts der PKV (WIP)}

\subsubsection{Mehrumsatzstudien}

Die Ausgabenstatistik der privaten Krankenversicherung spiegelt in drei Punkten nicht die volle Realität der werden. Eine Zuordnung der zahnärztlichen Ausgaben auf die Versicherten je nach Versicherungsstatus ist damit in der Ausgabenstatistik der privaten Krankenversicherung nahezu unmöglich.

Diese drei in der PKV-Ausgabenstatistik nicht befriedigend erfassten Punkte werden in den sogenannten Mehrumsatzstudien des Wissenschaftlichen Instituts der PKV (WIP) vollständig berücksichtigt. Selbstbehalte und Beihilfeleistungen sind hier explizit mit einbezogen worden. Methodisch kann zwischen Vollversicherung und Zusatzversicherung getrennt werden. Insgesamt heißt das: Mit den aus dem Risikostrukturausgleich der GKV (RSA) beziehungsweise PKV-Kopfstatistiken generierten Ergebnissen des Wissenschaftlichen Instituts der PKV lässt sich von 2001 bis einschließlich 2008 ein ergänzender, in mancherlei Hinsicht auch ein vervollkommnender Blick auf die Bedeutung der Privatpatienten für das Einkommen der Zahnärzte werfen. ${ }^{14}$

Die Vergütung der Zahnleistungen, die für Privatpatienten erbracht werden, unterscheidet sich von der Vergütung der Leistungen für Versicherte in

13 Der Frauenanteil unter den tätigen Zahnärzten nimmt zu. In Westdeutschland stieg die Frauenquote gegenüber dem Jahr 2000 um 5,4 Prozentpunkte auf 37,1 \%. In Ostdeutschland stieg die Frauenquote auf 58,5\%; vgl. Statistisches Jahrbuch 2009/2010, Bundeszahnärztekammer.

$14 \mathrm{Vgl}$. zu den Mehrumsatzstudien des Wissenschaftlichen Instituts des PKV (WIP) Niehaus, F. Weber, C. (2005); Niehaus, F. (2006); Niehaus, F. (2006); Niehaus, F. (2007); Niehaus, F. (2009); Niehaus, F. (2010). 


\begin{tabular}{|c|c|c|}
\hline & $\begin{array}{c}\text {... die } 30 \text { häufigsten GOZ- } \\
\text { Positionen als Ausgangs- } \\
\text { punkt }\end{array}$ & $\begin{array}{c}\text {... die } 30 \text { umsatzstärksten } \\
\text { GOZP-Positionen als Aus- } \\
\text { gangspunkt }\end{array}$ \\
\hline $\begin{array}{l}\text { Vergütungsunterschied mit } \\
\text { Berücksichtigung der Zahn- } \\
\text { reinigung }\end{array}$ & $+51,78 \%$ & $+63,23 \%$ \\
\hline $\begin{array}{l}\text { Vergütungsunterschied ohne } \\
\text { Berücksichtigung der Zahn- } \\
\text { reinigung }\end{array}$ & $+46,36 \%$ & $+58,94 \%$ \\
\hline
\end{tabular}

\begin{tabular}{|c|c|c|c|}
\hline & 2003 & 2007 & Veränderung in \% \\
\hline $\begin{array}{l}\text { Gesamtumsatz je Zahnarzt- } \\
\text { praxis, in } €\end{array}$ & $457.000 €$ & $462.000 €$ & $+1,1 \%$ \\
\hline $\begin{array}{l}\text { Einnahmen über KZV (Kassen- } \\
\text { praxis), in } €\end{array}$ & $265.000 €$ & $240.000 €$ & $-9,4 \%$ \\
\hline $\begin{array}{l}\text { Einnahmen aus der Privatliqui- } \\
\text { dation (Privatpraxis), in } €\end{array}$ & $192.000 €$ & $222.000 €$ & $+15,6 \%$ \\
\hline $\begin{array}{l}\text { Anteil der Privatliquidation am } \\
\text { Gesamtumsatz }\end{array}$ & $42,0 \%$ & $48,1 \%$ & $+14,5 \%$ \\
\hline
\end{tabular}

der GKV. Der sogenannte Mehrumsatz, der so oberhalb der gesetzlichen Vergütung durch Privatversicherte jährlich entsteht, betrug im Jahr 2008 insgesamt 10,86 Mrd. $€$. Davon entfielen allein 2,12 Mrd. $€$ auf Leistungen im zahnmedizinischen Bereich. Das entspricht einem Anteil von 19,5\%.

Zur Berechnung des Mehrumsatzes weist das Wissenschaftliche Instituts der PKV die Ausgaben der Beihilfestellen, der Selbstbehalte und der privaten Krankenversicherungen für zahnärztliche Leistungen mit 3,62 Mrd. € aus. Auf jeden Vollversicherten ${ }^{15}$ in der PKV entfielen damit im Durchschnitt $415 €$ für Zahnleistungen. Teilt man die Ausgaben der Selbstzahler, Beihilfestellen und privaten Krankenversicherungen für Arzthonorare in Höhe von 3,62 Milliarden auf die $63.532 \mathrm{im} \mathrm{Jahr}$ 2008 tätigen Zahnärzte ${ }^{16}$ auf, ergibt sich ein durchschnittlicher Umsatz von ca. $56.979 €$ je Zahnarzt. Im Jahr 2001 lag die Zahl der praktizierenden Zahnärzte noch bei 60.858 . Auf diese entfielen Ausgaben der Selbstzahler (Selbstbehalte), Beihilfe und PKV in Höhe von 2,79 Mrd. €. ${ }^{17}$ Damit ergibt sich ein durchschnittlicher Umsatz im

Um die Bedeutung der Einnahmen der Abrechnung über die GOZ zu erfassen, empfiehlt es sich, ergänzend zu analysieren, inwieweit sich das Vergütungsniveau von GOZ und BEMA unterscheidet. Einen derartigen Vergleich hat das Wissenschaftliche Institut der PKV unternommen. ${ }^{18}$ Ausgehend von den bei PKV-Unternehmen eingereichten Rechnungen der Privatversicherten nach GOZ wird berechnet, wie viel die Zahnärzteschaft an Einnahmen generiert hätte, wenn sie nicht nach der GOZ, sondern nach BEMA abgerechnet hätte. Die Datengrundlage der nach GOZ abgerechneten Leistungen bildet eine Stichprobe von anonymisierten zahnärztlichen Rechnungen von PKVVersicherten aus dem Jahr 2008. Diesen
Rechnungen sind neben den abgerechneten Gebührenpositionen auch die Steigerungssätze und die Rechnungsbeträge zu entnehmen. Dabei gilt es zu betonen, dass die zahnärztlichen Privatliquidationen für selbstzahlende GKV-Versicherte in den Daten nicht enthalten sind.

(a) Die häufigsten GOZ-Positionen als Ausgangspunkt

Wählt man als Ausgangspunkt einer Gegenüberstellung der Vergütungspositionen die 30 am häufigsten abgerechneten GOZ-Positionen, ordnet diesen Positionen - soweit möglich eine vergleichbare BEMA-Nummer zu und nimmt angesichts diverser Zuordnungsprobleme und Strukturunterschiede zwischen GOZ und BEMA begründete Bereinigungen vor, ${ }^{19}$ dann stellt man Folgendes fest: In der Rechnungsstichprobe stellten die Zahnärzte für die 30 am häufigsten abgerechneten GOZ-Positionen im Bereich der konservierenden/chirurgischen Behandlung und Zahnersatz im Jahr 2008 ca. 2,563 Mio. € in Rechnung. In der GKV wären es für die gleiche Leistung nach BEMA nur 1,699 Mio. € gewesen. ${ }^{20}$ Der PKV-Betrag liegt damit um knapp $52 \%$ über dem vergleichbaren Betrag der GKV.

(b) Die umsatzstärksten GOZ-Positionen als Ausgangspunkt ${ }^{21}$

Wählt man als Ausgangspunkt einer Gegenüberstellung der Vergütungspositionen die 30 umsatzstärksten GOZPositionen (= durchschnittlich ca. $60 \%$ des Umsatzes eines Zahnarztes aus der GOZ), ordnet diesen Positionen - soweit möglich - eine vergleichbare BEMA-Nummer zu und nimmt angesichts diverser Zuordnungsprobleme und Strukturunterschiede zwischen
15 Vollversicherte erhalten ihre Leistungen entweder zu 100 \% von der PKV oder zu Teilen von der Beihilfe und PKV.

16 Daten der Bundeszahnärztekammer 2010.

17 Angaben des Wissenschaftlichen Instituts der PKV (WIP).

18 Vgl. Niehaus, F.; Kessler, T.; Finkenstädt, V. (2011), Ein Vergleich der zahnärztlichen Vergütung nach GOZ und BEMA (erscheint in Kürze)

19 Findet sich keine vergleichbare Gebührenposition im BEMA, wird die Position beim Vergütungsvergleich nicht berücksichtigt.

20 Bei dem Vergleich wird der Punktwert der Ersatzkassen des Jahres 2008 verwendet. Damit wird auf der GKV-Seite (im Vergleich zu den Primärkassen) die relativ höhere Vergütung gewählt.

21 Vgl. Niehaus, F.; Kessler, T.; Finkenstädt, V. (2011), S. $11 \mathrm{ff.}$ 
Tab. 5: Aufwendungskosten und Reinerträge je Zahnarztpraxis

\begin{tabular}{|l|c|c|c|}
\hline & 2003 & 2007 & Veränderung in \% \\
\hline $\begin{array}{l}\text { Aufwendungen Zahnarztpraxis } \\
\text { in } €\end{array}$ & $314.000 €$ & $303.000 €$ & $-3,5 \%$ \\
\hline Reinertrag je Zahnarztpraxis in $€$ & $143.000 €$ & $159.000 €$ & $+11,2 \%$ \\
\hline Inflationsindex & 96,9 & 103,9 & $+7,2 \%$ \\
\hline \multicolumn{2}{|l}{ Quelle: u.a. Statistisches Bundesamt, Fachserie 2, Reihe 1.6.1 } \\
\hline
\end{tabular}

Tab. 6: Einnahmen je Inhaber einer niedergelassenen vertragszahnärztlichen Praxis

\begin{tabular}{|l|c|c|c|c|}
\hline & 2000 & 2003 & 2008 & $\begin{array}{c}\text { Veränderung } \\
\text { in \% }\end{array}$ \\
\hline $\begin{array}{l}\text { Gesamtumsatz je } \\
\text { Inhaber in } €\end{array}$ & $336.602 €$ & $355.038 €$ & $368.419 €$ & $+9,5 \%$ \\
\hline $\begin{array}{l}\text { Einnahmen über KZV } \\
\text { (Kassenpraxis), in } €\end{array}$ & $205.654 €$ & $207.697 €$ & $186.420 €$ & $-9,4 \%$ \\
\hline $\begin{array}{l}\text { Einnahmen aus der Pri- } \\
\text { vatliquidation (Privat- } \\
\text { praxis), in } €\end{array}$ & $131.948 €$ & $147.341 €$ & $181.999 €$ & $+37,9 \%$ \\
\hline $\begin{array}{l}\text { Anteil der Privatliquida- } \\
\text { tion am Gesamtumsatz }\end{array}$ & $39,2 \%$ & $41,5 \%$ & $49,4 \%$ & $+26,0 \%$ \\
\hline \begin{tabular}{l} 
Quelle: KZBV-Kostenstrukturerhebung (2010) \\
\hline
\end{tabular}
\end{tabular}

Tab. 7: Betriebsausgaben/Kosten und Reinerträge je Inhaber einer niedergelassenen vertragszahnärztlichen Praxis

\begin{tabular}{|l|c|c|c|c|}
\hline & 2000 & 2003 & 2008 & $\begin{array}{c}\text { Veränderung } \\
\text { in \% }\end{array}$ \\
\hline $\begin{array}{l}\text { Betriebsausgaben je } \\
\text { Inhaber in } €\end{array}$ & $239.980 €$ & $248.293 €$ & $251.101 €$ & $+4,6 \%$ \\
\hline $\begin{array}{l}\text { Ertrag/Überschuss je } \\
\text { Inhaber in } €\end{array}$ & $96.622 €$ & $106.745 €$ & $117.318 €$ & $+21,4 \%$ \\
\hline Inflationsindex & 92,7 & 96,9 & 106,6 & $+15,0 \%$ \\
\hline \multicolumn{2}{l|l}{ Quelle: KZBV - Kostenstrukturstatistik (2010) }
\end{tabular}

GOZ und BEMA begründete Bereinigungen vor, ${ }^{22}$ dann stell man fest, dass die Vergütung der GOZ $63 \%$ über der der BEMA liegt. ${ }^{23}$

\subsection{Ergebnisse auf Basis der Kostenstrukturstatistik des Statistischen Bundesamtes und der Kassenzahnärztlichen Bundesvereinigung}

\subsubsection{Ergebnisse je Zahnarztpraxis}

Als dritte und vierte Quelle werden im Folgenden Auswertungen des Statistischen Bundesamtes sowie Erhebungen der Kassenzahnärztlichen Bundesvereinigung zur Kostenstruktur in Zahnarztpraxen betrachtet. Aus den Daten des Statischen Bundesamtes geht hervor, dass eine durchschnittliche Zahnarztpraxis im Jahr 2007 einen Umsatz von $462.000 €$ erzielt hat. ${ }^{24}$ Auf Privatabrechnungen des Zahnarztes, die nicht über die KZV vereinnahmt wurden, entfiel hierbei ein Anteil von $48,1 \%$.

Vergleicht man die Daten von 2007 mit der vorangegangenen Erhebung des Statistischen Bundesamtes aus dem Jahr 2003, so zeigt sich, dass der Gesamtumsatz je Zahnarztpraxis nur moderat um $1,1 \%$ gestiegen ist. Dabei sind die Ein- nahmen aus der Kassenpraxis um fast $10 \%$ gesunken. Im Umkehrschluss heißt das, dass sich zur Kompensation der Einbußen in der GKV die Umsätze aus der Privatabrechnung - auch im Zuge der zunehmenden Bedeutung der gesetzlich versicherten Selbstzahler - deutlich erhöht haben. Die Einnahmen aus der Privatpraxis sind zwischen 2003 und 2007 um 15,6 \% von 192.000 auf $222.000 €$ gestiegen. Im Jahr 2003 betrug der Anteil der Rechnungen außerhalb der KZV-Vergütung am gesamten Umsatz noch 42,0 \%, im Jahr 2007 waren es schon $48,1 \%$.

Die Daten des Statistischen Bundesamtes erlauben auch einen Blick auf die Aufwendungen beziehungsweise Kosten der Zahnarztpraxen. Dabei werden unter anderem Aufwendungen für Personal, Material, fremde Laborkosten, Mieten und Fremdkapitalzinsen berücksichtigt. Es zeigt sich, dass die Kosten zwischen 2003 und 2007 in den Zahnarztpraxen - trotz einer Inflation von rund $7 \%$ - gesunken sind. Das gilt sowohl absolut als auch relativ. Als Anteil an den Einnahmen sind die Aufwendungen je Zahnarztpraxis zwischen den Jahren 2003 und 2007 von $68,8 \%$ auf $65,6 \%$ gesunken. ${ }^{25}$ Damit sind die Reinerträge - also die Einkommen - je Zahnarztpraxis deutlich gestiegen $(11,2 \%){ }^{26}$
22 Findet sich keine vergleichbare Gebührenposition im BEMA, wird die Position beim Vergütungsvergleich nicht berücksichtigt.

23 Vgl. Niehaus, F.; Kessler, T.; Finkenstädt, V. (2011), S. $15 \mathrm{ff}$.

24 Vgl. Statistisches Bundesamt (2006); Statistisches Bundesamt (2009); das Statistische Bundesamt kann noch keine Daten zur Kostenstrukturstatistik für das Jahr 2008 und 2009 zur Verfügung stellen.

25 Vgl. Taubmann, D.; Harrer, K. (2010); Hauschild, W.; Klose, A. (2006).

26 Zieht man die Summe der Aufwendungen von der Summe der Einnahmen ab, so erhält man den Reinertrag der Zahnarztpraxen. Der Reinertrag stellt nicht den betriebswirtschaftlichen Gewinn der Zahnarztpraxis dar, da u. a. Aufwendungen privater Natur für die Alters-, Invaliditäts-, Hinterbliebenen- und Krankenversicherung der Praxisinhaber und ihrer Familienangehörigen, auch Beiträge zu Versorgungseinrichtungen der Zahnärzte, nicht berücksichtigt werden. 
Tab. 8: Durchschnittliche Versicherten- und Kassenanteile an den Gesamtkosten bzw. BEMA- und GOZ-Honoraranteile an den Zahnarzthonoraren beim Zahnarzt nach der Festzuschusserhebung vdek (in Prozent)

\begin{tabular}{|c|c|c|c|c|}
\hline \multirow{2}{*}{\multicolumn{2}{|c|}{$\begin{array}{l}\text { Durchschnittliches Gesamthonorar } \\
\text { des Zahnarztes im Rahmen der Ver- } \\
\text { sorgung gesetzlich Versicherter }\end{array}$}} & 2005 & 2009 & Veränderung \\
\hline & & $699,00 €$ & $732,71 €$ & $+4,8 \%$ \\
\hline \multicolumn{5}{|l|}{ davon: } \\
\hline \multirow{2}{*}{ Honorar BEMA } & absolut in $€$ & $182,67 €$ & $174,63 €$ & $-4,4 \%$ \\
\hline & als Anteil in \% & $26,1 \%$ & $23,8 \%$ & $\begin{array}{l}-2,3 \%- \\
\text { Punkte }\end{array}$ \\
\hline \multirow[t]{2}{*}{ Honorar GOZ } & absolut in $€$ & $516,13 €$ & $558,07 €$ & $+8,1 \%$ \\
\hline & als Anteil in \% & $73,9 \%$ & $76,2 \%$ & $\begin{array}{l}+2,3 \%- \\
\text { Punkte }\end{array}$ \\
\hline \multicolumn{2}{|c|}{$\begin{array}{l}\text { Gesamtkosten (Arzthonorar + } \\
\text { Material + Labor) }\end{array}$} & $1.784,14 €$ & $1.885,93 €$ & $+5,7 \%$ \\
\hline \multicolumn{5}{|l|}{ davon: } \\
\hline \multicolumn{2}{|l|}{ Kassenanteil } & $40,7 \%$ & $38,0 \%$ & $\begin{array}{l}-2,7 \%- \\
\text { Punkte }\end{array}$ \\
\hline \multicolumn{2}{|c|}{ Selbstzahleranteil } & $59,3 \%$ & $62,0 \%$ & $\begin{array}{l}+2,7 \%- \\
\text { Punkte }\end{array}$ \\
\hline
\end{tabular}

Tab. 9: Versicherten- und Kassenanteile an den Gesamtkosten beim Zahnersatz nach einer Dokumentation der Spitzenverbände der GKV (2006) und vdek (2010), in Prozent

\begin{tabular}{|l|c|c|c|c|}
\hline & $2004^{*}$ & $2005^{*}$ & $2009^{* *}$ & $\begin{array}{c}\text { Veränderung } \\
\text { in \%-Punkten }\end{array}$ \\
\hline $\begin{array}{l}\text { Kassenanteil an den } \\
\text { Gesamtkosten }\end{array}$ & $58,1 \%$ & $42,6 \%$ & $38 \%$ & $-20,1$ \\
\hline $\begin{array}{l}\text { Selbstzahleranteil an } \\
\text { den Gesamtkosten }\end{array}$ & $41,9 \%$ & $57,4 \%$ & $62 \%$ & $+20,1$ \\
\hline \multicolumn{2}{l|}{ Quelle: ${ }^{*}$ GKV-Spitzenverbände (2006); ${ }^{* *}$ vdek (2010) } \\
\hline
\end{tabular}

\subsubsection{Ergebnisse je Inhaber einer niedergelassenen vertragszahnärztlichen Praxis}

Während sich die Daten des Statistischen Bundesamtes auf Größen je Zahnarztpraxis beziehen, nehmen die Erhebungen der Kassenzahnärztlichen Bundesvereinigung Bezug auf die Inhaber einer niedergelassenen vertragszahnärztlichen Praxis. ${ }^{27}$ Dabei liegen nicht nur Daten für die Jahre 2003 und 2007, sondern auch für den Zeitraum zwischen 2000 und 2008 vor. Trotz dieser und anderer definitorischer $\mathrm{Ab}$ weichungen bestätigen die Ergebnisse aus der Erhebung der Kassenzahnärzt- lichen Bundesvereinigung das Gesamtbild der empirischen Daten des Statistischen Bundesamtes. Dazu folgender Überblick:

Je Inhaber einer niedergelassenen vertragszahnärztlichen Praxis sind im Jahr 2008 Umsätze von durchschnittlich $368.419 €$ erzielt worden. Auf Privatabrechnungen entfiel hierbei ein Anteil von 49,4 \% des Umsatzes. Die Einnahmen, die nicht über die KZV vereinnahmt worden sind, machen damit auch nach Datenlage der Kassenzahnärztlichen Bundesvereinigung fast die Hälfte der Gesamteinnahmen des Zahnarztes aus.

Im Vergleich zum Jahr 2000 zeigt sich, dass der Gesamtumsatz je Inhaber einer vertragszahnärztlichen Praxis um 9,5\% gestiegen ist. Dabei sind die Einnahmen aus der Kassenpraxis um über $9 \%$ gesunken. Im Umkehrschluss heißt das, dass sich zur Kompensation der Einbußen im Rahmen der KZVVergütung die Umsätze aus der Privatabrechnung - auch im Zuge der zunehmenden Bedeutung der gesetzlich versicherten Selbstzahler - deutlich erhöht haben. Die Einnahmen aus der Privatpraxis sind zwischen 2000 und 2008 um fast $38 \%$ von rund 132.000 auf $182.000 €$ gestiegen. Im Jahr 2000 betrug der Anteil der Privatrechnungen am gesamten Umsatz noch 39,2 \%, im Jahr 2008 waren es schon 49,4\%.

Die Betriebsausgaben haben sich trotz einer Inflation von rund $15 \%$ mit $+4,6 \%$ unterdurchschnittlich entwickelt. Als Anteil an den Gesamteinnahmen sind die Aufwendungen je Inhaber einer niedergelassenen vertragszahnärztlichen Praxis zwischen den Jahren 2000 und 2008 sogar von $71,3 \%$ auf $68,2 \%$ gesunken. Implizit heißt das, dass sich die Überschüsse mit $+21,4 \%$ stets besser entwickelt haben als die Aufwendungen und Preise.

\section{Ursachen der Ausgabenentwicklung}

Die bisher dokumentierte (überproportionale) Dynamik der Privatliquidation im Zahnbereich lässt sich begründen. Im Interesse der Privatversicherten und gesetzlich versicherten Selbstzahler lohnt es sich deshalb, die Ursachen der Ausgaben- bzw. Umsatzentwicklung getrennt nach Selbstzahlereffekt, Preiseffekt, Struktur- und Mengeneffekt $\mathrm{zu}$ analysieren.

\subsection{Selbstzahlereffekte}

Seit 2005 gibt es in der GKV beim Zahnersatz keine prozentuale Erstattung auf die Behandlungskosten, sondern einen Festzuschuss je Indikation. Spätestens seit dieser - von seiner Wirkung her als Rationierung zu bezeichnenden - Umstellung hat sich die GKV im Bereich der Zahnleistungen zur

\footnotetext{
27 Vgl. KZBV - Kostenstrukturerhebung.

28 Vgl. vdek (2010), Festzuschusserhebung Folgeerhebung 2009.
} 
Teilkaskoversicherung entwickelt. Alle gesetzlich Versicherten sind anteilig mehr und mehr zum Selbstzahler geworden. Das hat entsprechende Auswirkungen auf die Zahlen zur Ausgabenentwicklung im Zahnbereich. Überall dort, wo sich die analysierten Größen nicht auf Privatliquidationen im engeren Sinne - nämlich Abrechnungen von Privatversicherten -, sondern auf Privatliquidationen von Privatversicherten und gesetzlich Versicherten beziehen, entstehen Zuwächse bei den GOZ-Honoraranteilen - ursächlich auch durch den vom Gesetzgeber im Zuge der Leistungsrationierung gewollten Bedeutungsverlust des Bewertungsmaßstabes zahnärztlicher Leistungen (BEMA). Eine Wirkung, die im folgenden »GKV-Selbstzahlereffekt « zu nennen ist.

Hinweise auf den GKV-Selbstzahlereffekt fanden sich schon in den Daten des Statistischen Bundesamtes und der Kassenzahnärztlichen Bundesvereinigung. Weitere empirische Anhaltspunkte dieses Effektes liefern darüber hinaus Beobachtungen mehrerer Studien aus der GKV. Exemplarisch seien hier Erhebungen des Verbandes der Ersatzkassen (vdek) aus dem Jahr 201028 und der Spitzenverbände der GKV aus dem Jahr $2006^{29}$ genannt. Beide Studien zusammengenommen zeigen, dass nicht nur der neue Festzuschuss zum Jahreswechsel 2004/2005 zu einem Anstieg des Selbstzahleranteils in der zahnmedizinischen Versorgung geführt hat, sondern auch zwischen 2005 und 2009 der Selbstzahleranteil der gesetzlich Versicherten kontinuierlich gewachsen ist.

Die Studie des Verbandes der Ersatzkassen bezieht sich auf eine Stichprobe im Jahr 2005 und 2009. Dabei hat jede teilnehmende Ersatzkasse qualitätsgesicherte Abrechnungsdaten aus Heilund Kostenplänen sowie Privatliquidationen erhoben. Die Stichprobe umfasst für den Leistungsbereich des Zahnersatzes die Bereiche der Regelversorgung sowie der gleich- und andersartigen Versorgung. Die Ergebnisse: Bei der Betrachtung der durchschnittlichen zahnärztlichen Honorare, die über den vertraglichen Leistungskatalog (BEMA) und die private Gebührenordnung (GOZ) abgerechnet werden, wird eine Verlagerung zum privaten Gebührenkatalog deutlich. ${ }^{30}$

Tab. 10: Häufigkeitsverteilung der Steigerungsfaktoren (bezüglich Anzahl der Leistungen und bezügl ich des Rechnungsbetrages) in \%

\begin{tabular}{|l|c|c|c|c|}
\hline & 2000 & 2009 & $\begin{array}{c}\text { Veränderung } \\
\text { in \%-Punkten } \\
\text { (2000-2009) }\end{array}$ \\
\hline $\begin{array}{l}\text { bezüglich der } \\
\text { Anzahl }\end{array}$ & $\begin{array}{c}\text { bis zum Regel- } \\
\text { höchstsatz }\end{array}$ & $87,8 \%$ & $71,1 \%$ & $-16,7$ \\
\cline { 2 - 5 } & $\begin{array}{c}\text { über dem Regel- } \\
\text { höchstsatz }\end{array}$ & $12,2 \%$ & $28,9 \%$ & $+16,7$ \\
\hline $\begin{array}{l}\text { bezüglich des } \\
\text { Rechnungsbe- } \\
\text { trages }\end{array}$ & $\begin{array}{c}\text { bis zum Regel- } \\
\text { höchstsatz }\end{array}$ & $67,8 \%$ & $47,0 \%$ & $-20,8$ \\
\cline { 2 - 5 } & $\begin{array}{c}\text { über dem Regel- } \\
\text { höchstsatz }\end{array}$ & $32,2 \%$ & $53,0 \%$ & $+20,8$ \\
\hline Quelle: Kessler, T. (2007); Kessler T. (2010) & & & \\
\hline
\end{tabular}

Im Jahr 2005 nahm im Rahmen der Versorgung gesetzlich Versicherter mit Zahnersatz das durchschnittliche BEMA-Honorar $(182,67 €)$ einen Anteil am Zahnarzthonorar von $26,1 \%$ ein und sank bis zum Jahr 2009 auf nur noch $23,8 \%$. Im Umkehrschluss lag der GOZ-Anteil am durchschnittlichen Gesamthonorar der Zahnärzte im Jahr 2009 bei durchschnittlich schon $76,2 \%$

Für die Gesamtkosten im Bereich des Zahnersatzes sind neben den Zahnarzthonoraren insbesondere auch Material- und Laborkosten verantwortlich. Betrachtet man deshalb nicht das Honorar des Zahnarztes, sondern die Gesamtkosten als Ausgangsgröße, so zeigt sich, dass seit 2005 der Selbstzahleranteil der gesetzlich Versicherten kontinuierlich von $59,3 \%$ auf $62 \%$ gewachsen ist. Das ist umso bedeutsamer, weil der Dokumentation der Spitzenverbände der GKV aus dem Jahre $2006 \mathrm{zu}$ entnehmen ist, dass schon im Zuge der Einführung des Festzuschusses zum Jahreswechsel 2004/2005 der Selbstzahleranteil von $41,9 \%$ auf $57,4 \%$ sprunghaft angestiegen ist. Führt man beide Studien zusammen, lässt sich damit folgende Aussage formulieren. Während im Jahr 2004 die Beziehung zwischen dem Kassen- und Selbstzahleranteil annähernd als Verhältnis 60 zu 40 beschreibbar war, haben sich die Bedingungen für den Selbstzahler in der GKV bis zum Jahr 2009 mehr als umgekehrt. Im Jahr 2009 mussten mehr als $60 \%$ der Gesamtkosten vom Versicherten selbst getragen werden.

\subsection{Preiseffekte}

Steigende Ausgaben durch einen Preiseffekt können entstehen, wenn ein Wert aus der in der GOZ beschriebenen Formel »Punktzabl $x$ einheitlicher Punktwert $=$ Gebührensatz $x$ Steigerungssatz = Honorar $"$ steigt. Die GOZ ist seit 1988 gültig. Die Punktzahl und der Punktwert der GOZ (5,62421 Cent) sind seitdem stabil geblieben. Lediglich die Häufigkeit des angewandten Steigerungssatzes liegt im Ermessen der Zahnärzte. Hier lässt sich - neben dem Selbstzahlereffekt - ein weiterer Grund für die wachsende Bedeutung der Privatpatienten für das Einkommen der Zahnärzte identifizieren:

Wenn man in der zahnärztlichen Versorgung die Häufigkeitsverteilung der Steigerungsfaktoren ab 2000 betrachtet, ist - von einem hohen Niveau kommend - eine deutliche Verschiebung der Steigerungsfaktoren in den Bereich jenseits des Regelhöchstsatzes zu erkennen. Die Anzahl der bis zum Regelhöchstsatz abgerechneten Leistungen sank von fast $88 \%$ auf $71 \%$ in 2009. Gleichzeitig stieg der Anteil der über dem Regelhöchstsatz abgerechneten Gebührenpositionen von $12,2 \%$ (2000) auf nunmehr fast $29 \% .31$

29 Vgl. Spitzenverbände der GKV (2006), Untersuchung der Auswirkungen befundbezogener Festzuschüsse.

30 Vgl. vdek (2010), S. 20 ff.

31 Vgl. Kessler, T. (2007); Kessler, T. (2010) Jahresberichte zur zahnärztlichen Versorgung. 
Deutlicher wird diese von den Zahnärzten direkt zu beeinflussende Verschiebung, wenn man die Häufigkeit der Anwendungen der Steigerungsfaktoren nicht auf die Anzahl, sondern auf den Rechnungsbetrag beziehungsweise den Umsatz bezieht. Hierbei würde sachlich richtig berücksichtigt, dass sich Verhaltensänderungen beim Steigerungsfaktor für den Zahnarzt insbesondere dann lohnen, wenn es sich um Leistungen handelt, die in der GOZ relativ hohe Punktwerte aufweisen. Das Ergebnis: Die Anzahl der bis zum Regelhöchstsatz abgerechneten Leistungen sank von 67,8 \% im Jahr 2000 auf 47,0 \% in 2009. Gleichzeitig stieg der Anteil der über dem Regelhöchstsatz abgerechneten Gebührenpositionen von 32,2\% (2000) auf nunmehr 53 \% im Jahr 2009.32 Das heißt, die Häufigkeit der Anwendung eines Steigerungsfaktors über dem Regelhöchstsatz hat sich (nach Umsatz) innerhalb von 9 Jahren um fast $65 \%$ erhöht.

\subsection{Struktur- und Mengeneffekte}

Die sich verändernde Anwendung der Steigerungsfaktoren »nach oben « führt $\mathrm{zu}$ einem starken Preiseffekt. Davon unabhängig sind auch Struktur- und Mengeneffekte zu betrachten:

- Ein Struktureffekt entsteht unter anderem durch Analogabrechnungen. Weil die GOZ seit 1988 nicht mehr novelliert worden ist, nehmen Analogabrechnungen (für die in der GOZ nicht erfassten neuen Leistungen) kontinuierlich zu. Das wiederum hat eine Anhebung des Honorarniveaus zur Folge, weil die Zahnärzte bei der Analogberechnung de facto die Freiheit haben, im Verhältnis zum Aufwand für die tatsächlich erbrachte Leistung zu hoch bewertete Gebührenpositionen heranzuziehen, und diese Möglichkeit auch nutzen.

- Mengeneffekte lassen sich dem Grunde nach auf zwei Tatbestände zurückführen. Erstens: Da Patienten häufig eine eingeschränkte Konsumentensouveränität aufweisen, können Ärzte durch Wiederbestellung von Patienten die Nachfrage nach ihren Leistungen (bedingt) selbst induzieren. Als Alternative zur
Wiederbestellung der Patienten ist zweitens denkbar, dass vom Zahnarzt medizinisch nicht notwendige Leistungen erbracht werden. Beide Sachverhalte entsprechen dem Tatbestand einer angebotsinduzierten Mengenausweitung.

Mengenausweitungen und Analogabrechnungen bieten sehr gute Möglichkeiten, durch die allgemeine Inflation entstandene reale Einkommensverluste auszugleichen. Diese Möglichkeiten das zeigen folgende empirische Daten sind in der Vergangenheit mehr als überproportional zur Preisentwicklung genutzt worden.

Struktur- und Mengeneffekte lassen sich in Rechnungsauswertungen empirisch nicht »sauber « trennen. In der direkten Konsequenz sind alle nun folgenden Ergebnisse der repräsentativen Rechnungsauswertung des PKV-Verbandes immer sowohl als deutliche Hinweise auf Struktur- als auch Mengeneffekte zu betrachten.

- Durchschnittlicher Rechnungsbetrag Der durchschnittliche Rechnungsbetrag bezieht sich auf die vom Versicherten beim Versicherungsunternehmen eingereichten Rechnungen. Der durchschnittliche Rechnungsbetrag ist als arithmetisches Mittel des Rechnungsendbetrages dargestellt.

Die (eingereichten) Rechnungsbeträge sind in der privaten Krankenversicherung im Durchschnitt erheblich gestiegen. Während die Inflation zwischen 1999 und 2009 nur bei 17 \% lag, sind die Vergütungshöhen je Rechnung um fast $144 \%$ angestiegen. Damit sind die durchschnittlichen Rechnungsbeträge $\quad 8,4 \mathrm{mal}$ schneller gewachsen als die Preisentwicklung, die jährlich vom Statistischen Bundesamt erfasst und dokumentiert wird.

Tab 11: Entwicklung des durchschnittli-

chen Rechnungsbetrages, zahnmedizinische Leistungen (in \%)

\begin{tabular}{|l|c|c|c|c|}
\hline & 1999 & 2005 & 2009 & $\begin{array}{c}\text { An- } \\
\text { stieg } \\
\text { in \% }\end{array}$ \\
\hline $\begin{array}{l}\text { Rech- } \\
\text { nungs- } \\
\text { betrag }\end{array}$ & $505,1 €$ & $864,0 €$ & $1.231,9 €$ & $\begin{array}{c}+143,9 \\
\%\end{array}$ \\
\hline $\begin{array}{l}\text { Inflations- } \\
\text { index }\end{array}$ & 91,4 & 100 & 107 & $+17,1 \%$ \\
\hline $\begin{array}{l}\text { Quelle: PKV-Verband (2010), Ergebnisse der Rech- } \\
\text { nungsauswertung }\end{array}$ & & & & \\
\hline
\end{tabular}

- Betrag je Zahnarztkontakt/Praxiskontakt/Behandlungstag

Der Betrag je Zahnarztkontakt (Praxiskontakt) - korrekt als Betrag je Behandlungstag bezeichnet - ist diejenige abgerechnete Leistung in $€$, die auf einer zur Kostenerstattung eingereichten Rechnung während eines Behandlungstages vom Zahnarzt erbracht worden ist (= Summe der Rechnungspositionsbeträge, das heißt zum Beispiel ohne Zabntechnik, Auslagen oder Wegegeld). Jeder Behandlungstag entspricht dabei einem Zahnarztkontakt, wobei in diesem Zusammenhang - sachlich richtig - sowohl Zahnarztkontakte im Sinne einer persönlichen Begegnung zwischen Zahnarzt und Patient als auch Praxiskontakte obne direkte Zahnarztbegegnung zu interpretieren sind.

Die Betrachtung des Betrages je Zahnarztkontakt zeigt folgendes Ergebnis: Während die Preise seit 1999 »nur « um rund $17 \%$ angestiegen sind, lag das Wachstum der Euro-Beträge je Zahnarztkontakt/Behandlungstag bei der zahnärztlichen Versorgung bei über $87 \%$. Damit sind die je Behandlungstag eingereichten Leistungen 5,1mal schneller gewachsen als die Preisentwicklung.

\section{Tab. 12: Betrag je Arztkontakt/Praxis-}

kontakt/Behandlungstag

(Zahn, ohne Zahntechnik), in €

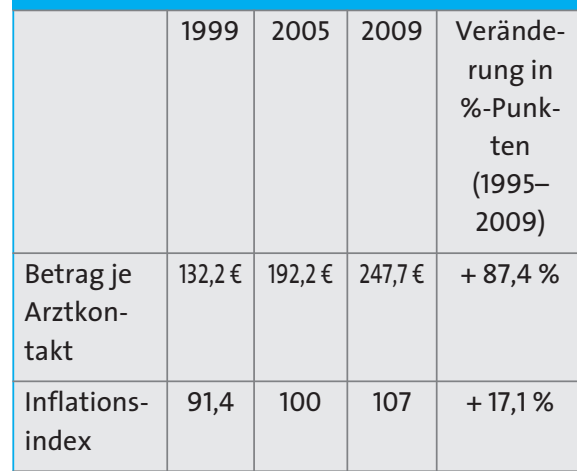

Quelle: PKV-Verband ( 2010), Ergebnisse der Rechnungsauswertung

32 Vgl. Kessler, T. (2007); Kessler, T. (2010) Jahresberichte zur zahnärztlichen Versorgung.

33 Vgl. PKV-Verband (2010), Ergebnisse der Rechnungsauswertung. 
- Betrag je Einzelleistung/Gebührenposition

Weitere Hinweise auf existierende Struktur- und Mengeneffekte liefert die Entwicklung des Betrages je Einzelleistung/Gebührenposition. Der Betrag je Einzelleistung ist die Summe der Rechnungspositionsbeträge je abgerechnete Gebührenposition. Mehrfachabrechnungen einer Gebührenposition werden dabei berücksichtigt.

Während die Inflation seit 1999 bei gut $17 \%$ lag, sind die Beträge je Einzelleistung beziehungsweise je Gebührenposition des Zahnarztes auf über $47 \%$ angestiegen. Damit sind die Beträge je Einzelleistung 2,8mal schneller gewachsen als die Preisentwicklung.

\section{Tab. 13: Beträge je Einzelleistung,}

zahnmedizinische Leistungen (in €)

\begin{tabular}{|l|c|c|c|c|}
\hline & 1999 & 2005 & 2009 & $\begin{array}{c}\text { An- } \\
\text { stieg } \\
\text { in \% }\end{array}$ \\
\hline $\begin{array}{l}\text { Betrag je } \\
\text { Leistung }\end{array}$ & 12,2 & 15,6 & 18,0 & $\begin{array}{c}+47,5 \\
\%\end{array}$ \\
\hline $\begin{array}{l}\text { Inflations- } \\
\text { index }\end{array}$ & 91,4 & 100 & 107 & $\begin{array}{c}+17,1 \\
\%\end{array}$ \\
\hline
\end{tabular}

Quelle: PKV-Verband ( 2010), Ergebnisse der Rechnungsauswertung

\section{Zusammenfassung und Fazit}

Im vorliegenden Aufsatz zur Ausgabenbeziehungsweise Umsatzentwicklung in der zahnärztlichen Versorgung belegen alle verfügbaren Daten - von der Ausgabenstatistik der PKV bis hin zur Kostenstrukturstatistik der Kassenzahnärztlichen Bundesvereinigung, dass die Bedeutung der Privatpatienten für den Umsatz und damit für das Einkommen der Zahnärzte wächst. Die dabei unter anderem von den Leistungserbringern selbst verursachte überproportionale Ausgaben- und Umsatzdynamik muss sowohl im Interesse der Privatversicherten, der gesetzlich versicherten Selbstzahler als auch der Zahnärzteschaft gestoppt werden. Einige Ergebnisse im Überblick:

- In der PKV sind zwischen 1999 und 2009 im Bereich der zahnmedizinischen Versorgung die Ausgaben je Versicherten um $42 \%$ angestiegen. Im gleichen Zeitraum lag die allgemeine Preisentwicklung bei lediglich $17,1 \%$.
- Die Pro-Kopf-Ausgaben der Privatversicherten im Bereich der zahnmedizinischen Versorgung bezifferten sich 2009 auf 487,6 $€$. Die durchschnittlichen Ausgaben der GKV lagen dagegen - auch weil viele Leistungskürzungen stattgefunden haben - bei nur 160,2 $€$.

- Zwischen 2001 und 2009 ist der durchschnittliche Umsatz durch Privatversicherte je Zahnarzt um 41,7 $\%$ angestiegen. Die allgemeine Preisentwicklung lag bei lediglich 13,2\%.

- Die Vergütung der Leistungen, die für Privatpatienten erbracht werden, unterscheidet sich von der Vergütung der Leistungen für Versicherte in der GKV. Der Mehrumsatz, der so durch Privatversicherte jährlich entsteht, betrug $2008 \quad 10,9$

Mrd. €. Davon ent-

fielen allein 2,1 Mrd. $€$ auf Leistungen im Zahnbereich. Das entspricht einem Anteil von 19,3\%.

- Eine Analyse der 30 umsatzstärksten GOZ-Gebührenpositionen (= durchschnittlich ca. $60 \%$ des Umsatzes eines Zahnarztes aus der GOZ) zeigt, dass deren Vergütungswert $63 \%$ über dem vergleichbaren GKV- beziehungsweise BEMA-Wert liegt.

- Nach Angaben der KZBV sind zwischen 2000 und 2008 die Umsätze je Inhaber einer vertragszahnärztlichen Praxis um 9,5\% angestiegen. Der Umsatz aus der Privatabrechnung hat sich dagegen mit $+37,9 \%$ überproportional entwickelt. Damit machen die Einnahmen außerhalb der KZV-Vergütung fast $50 \%$ der Gesamteinnahmen des Zahnarztes aus.

Schon diese Ergebnisausschnitte aus der Volldokumentation zeigen, dass es keinen Nachholbedarf bei der Honorierung privatzahnärztlicher Leistungen gibt. Als Ursache für die beobachteten Ausgaben- respektive Einnahmenentwicklungen sind Selbstzahler-, Preis-, Struktur- und Mengeneffekte gefunden worden.

- Selbstzahlereffekt: Zwischen 2004 und 2009 ist der Selbstzahleranteil gesetzlich Versicherter (mit eventueller privater Zahnzusatzversicherung) an den Gesamtkosten der zahnmedi- zinischen Versorgung im Bereich des Zahnersatzes - auch, aber nicht nur durch das Festzuschusssystem - von ca. $42 \%$ auf $62 \%$ angestiegen. Das in den Gesamtkosten enthaltene Zahnarzthonorar ist dabei (mit steigender Tendenz) zu einem Anteil von über $76 \%$ als GOZ-Honoar abgerechnet worden.

- Preiseffekt: Die Häufigkeit der Anwendung eines Steigerungsfaktors über dem Regelhöchstsatz hat sich (nach Umsatz) innerhalb von 9 Jahren um fast $65 \%$ erhöht.

Vor dieser unter Umständen überfordernden Entwicklung müssen die Versicherten schon im Sinne des geplanten
Patientenrechtgesetzes
geschützt werden.

- Mengen- und Struktureffekt: Während die allgemeine Preisentwicklung seit 1999 um 17 \% angestiegen ist, lag das Wachstum der Euro-Beträge je Zahnarztkontakt bei über $87 \%$, die Entwicklung der Beträge je Gebührenposition bei 47,5\% und der Anstieg des durchschnittlichen Rechnungsbetrages bei stattlichen $144 \%$.

Die Ursachen für den Ausgaben- bzw. Umsatzanstieg sind damit komplex. In der Summe führt der Anstieg zu einer erheblich wachsenden Bedeutung der Privatpatienten und Selbstzahler für das Einkommen der Zahnärzte. Die zunehmende Dynamik bezahlen die gesetzlich versicherten Selbstzahler direkt aus ihrem Einkommen, Privatversicherte indirekt über entsprechend steigende Versicherungsbeiträge. Vor dieser unter Umständen überfordernden Entwicklung müssen die Versicherten in Deutschland schon im Sinne des im Jahr 2011 geplanten Patientenrechtgesetzes geschützt werden. Denn zum Patienten- und Versichertenschutz, zur Stärkung der Patientenautonomie, gehört zwingend auch die Sicherung einer qualitativ hochwertigen zahnmedizinischen Versorgung zu angemessenen Preisen.

Die zu schützenden Versicherten das kann nicht häufig genug betont 
werden - sind im Fall der Novellierung der Gebührenordnung für Zahnärzte (GOZ) 8,8 Mio. Privatversicherte in der Krankenvollversicherung, 4,2 Mio. Beihilfeberechtigte, 12,4 Mio. privat zahnärztlich Zusatzversicherte mit einem GKV-Grundschutz, 0,9 Mio. Mitglieder in der Postbeamtenkrankenkasse/Krankenversorgung der Bundesbahnbeamten und fast 58 Mio. gesetzlich versicherte Selbstzahler. Denn während es Privatversicherte von seither gewohnt sind, zahnärztliche Leistungen in der Regel vollständig nach der GOZ abzurechnen, spüren gesetzlich Versicherte im Zuge von Rationierungen erst in den letzten Jahren, dass sie beim Zahnarzt mehr und mehr als Selbstzahler auftreten (müssen). Sie erhalten im zunehmenden Maße (beim Zahnersatz zu 3/4) eine Abrechnung auf Basis der GOZ. Das heißt implizit, dass eine ausgewogene Weiterentwicklung der GOZ 100 \% der Patienten in Deutschland etwas angeht; sie ist nicht nur im Interesse der Privatversicherten und der Beihilfe, sondern im Interesse aller.
Literatur:

Bundeszahnärztekammer (2010), Statistisches Jahrbuch 2009/2010.

General Anzeiger (2010), Ich bin Anwalt der Versicherten, 20.3.2010.

GKV-Rechnungsergebnisse (endgültig), 1999 bis 2009.

Hauschild, W.; Klose, A. (2006), Kostenstrukturen bei Arzt- und Zahnarztpraxen 2003, in: Statistisches Bundesamt, Wirtschaft und Statistik 11/2006.

Kessler, T. (2007), Jahresbericht zahnärztliche Versorgung - Auswertung von Rechnungsdaten 2004/2005/2006, WIP-Berichterstattung (unveröffentlicht).

Kessler, T. (2010), Jahresbericht zahnärztliche Versorgung - Auswertung von Rechnungsdaten 2007/2008/2009, WIP-Berichterstattung (unveröffentlicht).

Kassenzahnärztliche Bundesvereinigung (2010), KZBV - Kostenstrukturerhebung.

Niehaus, F.; Weber, C. (2005), Der überproportionale Finanzierungsbeitrag privat versicherter Patienten im Gesundheitswesen, WIP-Projektpapier.

Niehaus, F. (2006), Der überproportionale Finanzierungsbeitrag privat versicherter Patienten im Jahr 2004, WIP-Diskussionspapier 1/2006

Niehaus, F. (2006), Der überproportionale Finanzierungsbeitrag privat versicherter Patienten im Jahr 2005, WIP-Diskussionspapier 2/2006.

Niehaus, F. (2007), Der überproportionale Finanzierungsbeitrag privat versicherter Patienten im Jahr 2006, WIP-Diskussionspapier 9/2007.

Niehaus, F. (2009), Der überproportionale Finanzierungsbeitrag privat versicherter Patienten im Jahr 2007, WIP-Diskussionspapier 8/2009.

Niehaus, F.; Kessler, T.; Finkenstädt, V. (2011), Ein Vergleich der zahnärztlichen Vergütung nach GOZ und BEMA, WIP - Diskussionspapier, (erscheint in Kürze).

Niehaus, F. (2010), Der überproportionale Finanzierungsbeitrag privat versicherter Patienten im Jahr 2008, WIP-Diskussionspapier 5/2010.

PKV-Verband (2010), Ergebnisse der Rechnungsauswertung.

PKV-Zahlenberichte, $1985 / 1986$ bis 2009/2010.

Spitzenverbände der GKV (2006), Untersuchung der Auswirkungen befundbezogener Festzuschüsse, Dokumentation, 1.3.2006.

Statistisches Bundesamt (2010), Verbraucherpreise, abgerufen unter: www.destatis.de.

Statistisches Bundesamt (2006), Unternehmen und Arbeitsstätten - Kostenstruktur bei Arzt-, Zahnarzt- und Tierarztpraxen 2003, Fachserie 2, Reihe 1.6.1.

Statistisches Bundesamt (2009), Unternehmen und Arbeitsstätten - Kostenstruktur bei Arzt- und Zahnarztpraxen, Praxen von psychologischen Psychotherapeuten sowie Tierarztpraxen 2007, Fachserie 2, Reihe 1.6.1.

Taubmann, D.; Harrer, K. (2010), Kostenstrukturen bei Arzt- und Zahnarztpraxen, in: Statistisches Bundesamt, Wirtschaft und Statistik, 4/2010.

Vdek (2010), Festzuschuss-Erhebung - Erhebung zu den Auswirkungen befundbezogener Festzuschüsse beim Zahnersatz, Folgeerhebung 2009.

Welt (2009), Ich bin nicht den Ärzten verpflichtet, 28.11.2009. 